\title{
Experimental evaluation of light sword lens performance with a variable pupil size
}

\author{
W. Torres-Sepúlveda, ${ }^{* 1}$ A. Mira-Agudelo, ${ }^{1}$ J.F. Barrera, ${ }^{1}$ and A. Kolodziejczyk ${ }^{2}$ \\ ${ }^{1}$ Grupo de Óptica y Fotónica, Instituto de Física, Facultad de Ciencias Exactas y Naturales, Universidad de \\ Antioquia UdeA, Calle 70No. 52-21, Medellín, Colombia, \\ ${ }^{2}$ Faculty of Physics, Warsaw University of Technology, Koszykowa 75, 00-662 Warszawa, Poland.
}

Received May 24, 2018; accepted June 13, 2018; published June 30, 2018

\begin{abstract}
This paper presents an experimental study designed to tes the performance of a light sword lens (LSL) with different pupil sizes. To do so, Snellen optotype images obtained by a monofocal lens either with or without an LSL, were compared. Images were obtained for three different pupil sizes at several target vergences. The correlation coefficient and through-focus curves were obtained and compared. The experimental results show differences in the contrast and the depth of focus with different pupil sizes using a monofocal lens without an LSL. In contrast, when using a monofocal lens in combination with an LSL, the quality of images is similar for all pupils and target vergences used, with slight differences only in halos and contrast.
\end{abstract}

The light sword lens (LSL) is a refractive version of the diffractive light sword optical element which main feature is providing an extended depth of focus [1]. This lens is an axial asymmetric element and its focusing geometry consists of assigning different optical powers to adjacent infinitesimal angular sectors, in order to obtain angular modulation of optical power [2]. Theoretically, the light modulation produced by the LSL is independent of the pupil size because of its focusing geometry. Thus, with any illumination diameter, all angular sectors contribute to light modulation and depth of focus $\Delta f$. This effect is shown in Fig. 1.

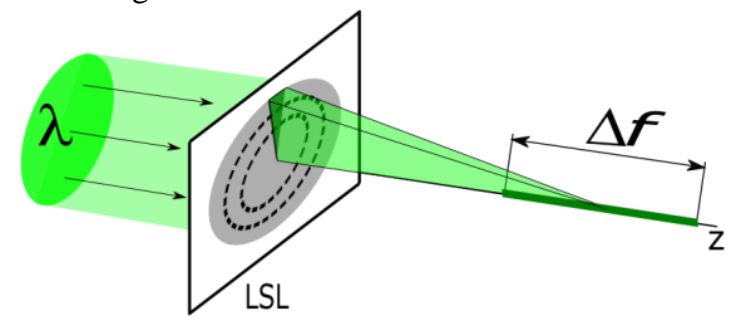

Fig. 1. Focusing geometry provided by the LSL when it is illuminated with light of a wavelength $\lambda$. For any pupil size (black dashed circumferences) all angular sectors corresponding to all optical powers contribute to light modulation.

Others have studied this hypothesis using computational simulations. They found that the quality of the images formed by the LSL does not change significantly with

\footnotetext{
*E-mail: walter.torres@udea.edu.co
}

different pupil sizes in contrast to other optical elements, like bifocal lenses or axicons [3].

Nevertheless, currently there are no reports of studies that experimentally verify this hypothesis, i.e., optical bench experiments or tests with real subjects.

Thus, this study was designed to analyze the performance of the LSL by using various pupil sizes.

We specifically evaluated three different pupil diameters in order to illustrate their impact on output images.

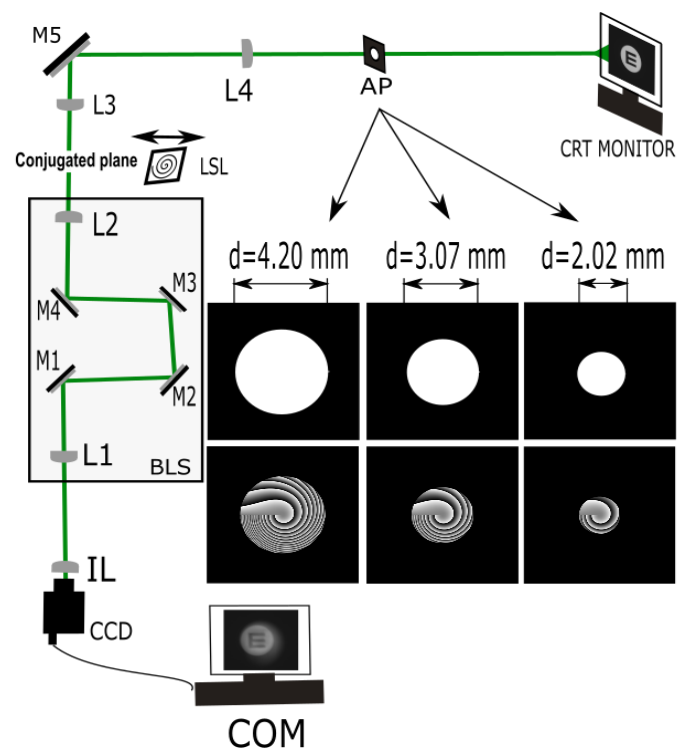

Fig. 2. Experimental setup for recording images of the Snellen optotype. L: lens, M: mirror, IL: imaging lens; CCD: CCD camera; COM: computer; BLS: Badal lens system; LSL: light sword lens and AP: artificial pupil.

Figure 2 shows the experimental set-up used in this work, similar to the monocular visual simulator described in detail in Ref. [4]. The experimental set-up permits different target vergences to be generated using a Badal lens system (BLS) for imaging without changing the angular size of targets. Also, the set-up allows the effective pupil size of the system to be controlled, which is a crucial feature in the present work. This control is 
Table 1: Experimental images of the Snellen optotype obtained with a monofocal lens without and with the light sword lens for three pupils with different sizes.

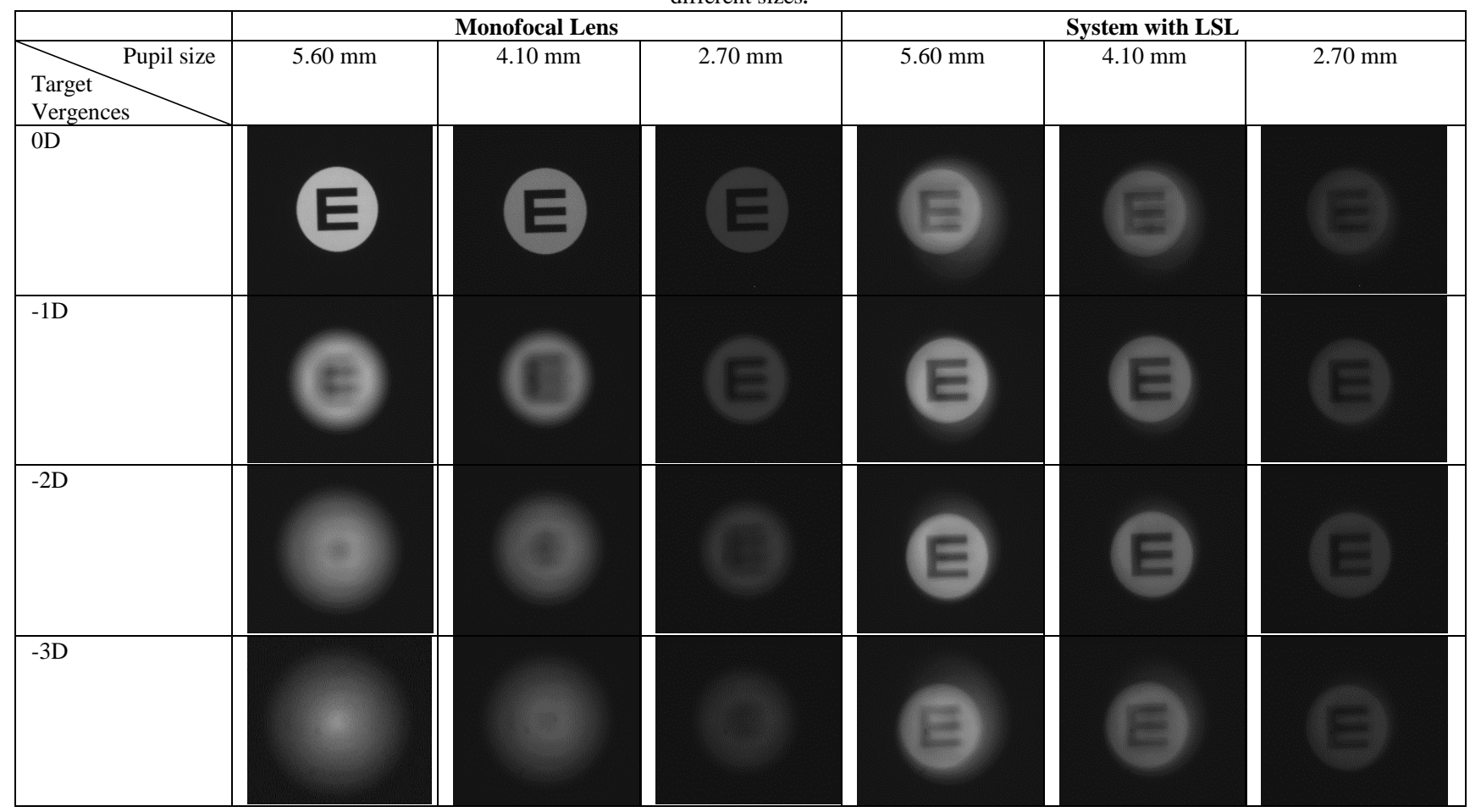

made with the artificial pupil (AP). Additionally, the system has a polychromatic stimuli pathway (green line in Fig. 2) for projecting targets like Snellen optotypes on a CRT monitor. The LSL is placed at a plane optically conjugated to the planes of the imaging lens (IL) and the AP (see Fig. 2). There is a magnification of $1.33 \times$ in the direction AP to IL. For this reason, the illuminated area at the LSL and the IL planes is 1.33 times the physical size of the AP. In this experiment, three circular AP were used with diameters of 2.02, 3.07 and $4.20 \mathrm{~mm}$ corresponding to sizes of $2.70,4.10$ and $5.60 \mathrm{~mm}$, respectively, at the LSL and IL planes.

The LSL was designed with an optical power range of $[-3,0] \mathrm{D}$. It has a diameter of $8 \mathrm{~mm}$, which means that the maximum pupil size was controlled only by the AP.

The experiment consisted of recording images of a Snellen optotype at six target vergences, from -4 to $+1 \mathrm{D}$ in steps of $1 \mathrm{D}$. The optotype had an angular size of 90 arc minutes, with a detail of 18 arc minutes. Images were obtained with a $75 \mathrm{~mm}$ focal length imaging lens and with the LSL located at the indicated conjugated plane (Fig. 2), operated as a contact lens put on the IL. The same measurements were made for three different artificial pupils; therefore, it was possible to compare the images corresponding to different target vergences and pupil sizes for the monofocal lens without (only the IL) and with the addition of the LSL.
Table 1 shows the recorded images formed by the monofocal lens adjusted at infinity without and with the addition of the LSL for different pupil sizes (columns) and target vergences (rows), covering the LSL's designed range of optical power, thus $[-3,0] \mathrm{D}$.

Images obtained by the monofocal lens show a similar trend for all pupils, with a progressive blurring for bigger target vergences. Also, there is a better contrast for the biggest pupil because more light is entering the system. Additionally, as anticipated, the images formed with the smaller pupil size present a larger depth of focus responsible for better quality of defocused images.

On the other hand, the images formed with the addition of the LSL exhibit different properties. Apart from contrast, the quality of the output images does not differ substantially for the target vergences and pupil sizes studied. All images formed by means of the LSL shown in Table 1 are recognizable.

Similar to the measurements obtained using the monofocal system without LSL, there is an incremental reduction in contrast as the pupils decrease in size. Additionally, characteristic halos produced by the LSL become more visible for larger pupils, which could slightly affect the output images.

An objective comparison of images from Table 1 can be made with the correlation coefficient (CC), calculated using the Matlab ${ }^{\circledR}$ software (The Mathworks Inc., Boston, MA, USA), defined as follows [5]: 


$$
r=\frac{\sum_{m} \sum_{n}\left(A_{m n}-\bar{A}\right)\left(I_{m n}-\bar{I}\right)}{\sqrt{\left(\sum_{m} \sum_{n}\left(A_{m n}-\bar{A}\right)^{2}\right)\left(\sum_{m} \sum_{n}\left(I_{m n}-\bar{I}\right)^{2}\right)}}
$$

where $\bar{A}$ and $\bar{I}$ are the average values of the images to be correlated, and $A_{m n}$ and $I_{m n}$ are image values at the pixel coordinate $(m, n)$. The reference image $I$ was obtained with the monofocal lens adjusted at infinity, using a $5.6 \mathrm{~mm}$ pupil at target vergence of $0 \mathrm{D}$, while $A$ corresponds to the images obtained for different pupils and vergences. All images were centered before the $\mathrm{CC}$ was calculated.

Figure 3(a) shows the behavior of the $\mathrm{CC}$ as a function of target vergence for the monofocal lens with all pupils; while Fig. 3(b) presents the CC curves corresponding to the system with the LSL.
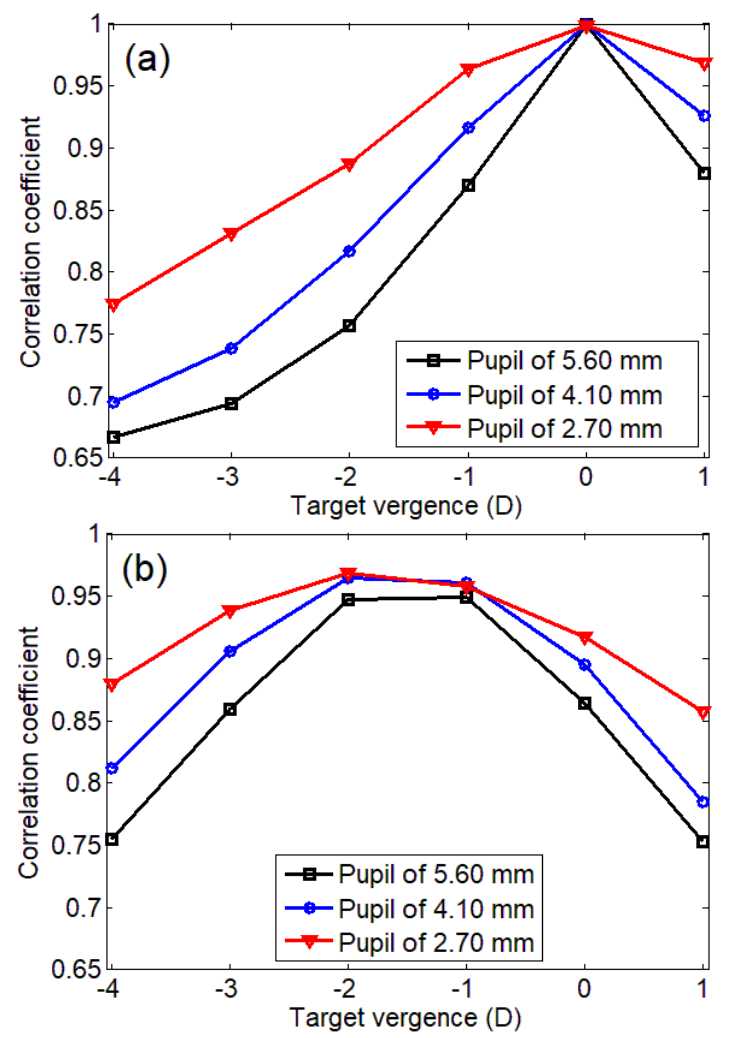

Fig. 3. Correlation coefficient as a function of target vergences, calculated from the images in Table 1. With (a) the monofocal lens and (b) the addition of the LSL.

Figure 3(a) shows the expected decrease of the CC when the absolute value of the target vergence increases, which is observed experimentally as progressive blurring of the output images. The CC has higher values for smaller pupils increasing the depth of focus and therefore improving the quality of images.

In Fig. 3(b), it is possible to note that for each pupil size the CC curve has a similar behavior, with higher CC values at the target vergences of -2 and $-1 \mathrm{D}$, and a decreasing trend for other target vergences. When using small pupils, the effect of halos is less noticeable and therefore the $\mathrm{CC}$ values are higher than those obtained with larger pupils.

The comparison of Fig. 3(a) and 3(b) reveals that when the LSL is used, the obtained CCs are higher than those obtained without the LSL in the target vergence range of $[-4,-1] \mathrm{D}$. The only exception is the case corresponding to the smallest aperture at vergence $-1 \mathrm{D}$, when the obtained CC values are similar for both the monofocal lens without and with the LSL.

However, for target vergences from 0 to $+1 \mathrm{D}$, the monofocal lens provides better CCs compared with the LSL, regardless of the pupil size.

The presented experimental results confirm that the extended depth of focus achieved by the LSL, does not exhibit significant variations when three pupils with different diameters are used.

It was found that for small, medium, and large pupils, the CC curves generated from the LSL system are similar for all evaluated target vergences. Nevertheless, they present a slight deviation due to the differences in the contrast of images, similar to the monofocal system. These findings reinforce the assumption that the LSL can be used for presbyopia correction in the form of contact or intraocular lenses. 'The behaviour of the human pupil is dynamic, constantly responding to the changing environment by adjusting its size; therefore, the independence of the aperture diameter in the LSL system shown in this paper could be particularly useful when developing visual aids.

According to Fig. 3 (b), the CC curves decline at the target vergence $0 \mathrm{D}$. Therefore, the LSL could perform worse for distance vision. This result coincides with our former subjective experiments presented in Ref. [4]. Thus, an effective solution to this problem demands a redesign of the element. The profile of the LSL defined by the linear angular function [4] can be modified to improve the correction for distance vision $(0 \mathrm{D})$, preserving angular modulation of optical power.

This research was made possible by grants from Universidad de Antioquia UdeA. Walter TorresSepúlveda acknowledges the support of Fondo Francisco José de Caldas (Colciencias-Colombia, grants 727 of 2015).

\section{References}

[1] G. Mikula, Z. Jaroszewicz, A. Kolodziejczyk, K. Petelczyc, M. Sypek, Opt Expr. 15, 9184 (2007).

[2] A. Kolodziejczyk, S. Bará, Z. Jaroszewicz, M. Sypek, J. Mod Opt. 37, 1283 (1990).

[3] K. Petelczyc et al., Photon. Lett. Poland 1, 55 (2009).

[4] A, Mira-Agudelo et al., Invest. Ophthalmol. Vis. Science 57, 6871 (2016)

[5] R.A. Fisher, Statistical Methods for Research Workers (New York, Hafner 1958). 